\title{
Reviews
}

\section{What You Should Do to Prepare for and Respond to Chemical, Radiological, Nuclear, and Biological Terrorist Attacks}

\author{
by Lynn E. Davis, Tom LaTourrette, David Mosher, Lois Davis
} and David Howell

Rand Corporation (2004): available at www.rand.org/publications/MR/MR1731.2/ (68 pages, free download)

and

\section{Hitting America's Soft Underbelly: The Potential Threat of Deliberate Biological Attacks Against the U.S. Agricultural and Food Industry by Peter Chalk}

Rand Corporation (2004): available at www.rand.org/publications/MG/MG135/ (65 pages, free download)

\section{Reviewed by Robert Stokes}

These two reviews offer a bit of a departure from traditional book reviews. It has been often noted among recent reviews of security-related publications here and in other venues that the recent speed of advancements (on both the sides of good and bad) in the security field has made their assessment problematic. This relates to nearly every facet of the field: from technology, to tactics, to legal issues - and especially, the ever-changing public policy arena. Thus, I have chosen to examine two publications that appear on the website of the Rand Corporation's 'Terrorism and Homeland Security' research area (at www.rand.org/research_areas/terrorism). Not to besmirch the many fine publishers and authors who work in the area of security and homeland security, but these reviews offer instead an assessment of foundation- and government-supported research publications in the area of security.

The first of the two chosen publications is entitled: What You Should Do to Prepare for and Respond to Chemical, Radiological, Nuclear, and Biological Terrorist Attacks. This is organized into a full report (funded by the Alfred P. Sloan Corporation) and a handy pocket-sized 'survival guide' edition. Both of these products are based on the power of the individual to make rational decisions that would serve to reduce the harm of a catastrophic nuclear, chemical, radiological or biological attack. This study and the subsequent report aim to fill a gap in response planning that has heretofore been dependent on coordinated state action. The authors note that these researchdriven survival strategies are intended to complement public efforts at disaster planning, not compete with them.

The work offers a series of terror scenarios - and they are catastrophic indeed: chemical exposure to sarin or hydrogen cyanide released into an office HVAC system; radiological exposure to 
americium 241 or cesium 137; nuclear exposure through the ground burst of a one-megaton nuclear device; and biological exposure to aerosolized anthrax or smallpox contagion released over a medium-sized city. After these scenarios are developed, the authors next identify the most efficacious responses related to each exposure. Potential actions were derived from an analysis of over 300 federal, state and locally developed guidelines. The researchers then ran the probable individualized terror response actions through the procedures expressed in model guidelines. These models controlled both for the type and location of the attack and for the individuals' relative distance from the hypothesized event. Finally, the recommendations were vetted by focus groups to assess their limitations with regard to a number of factors, including cost, ease of use, continuity with expert recommendations, etc.

Chapter Three of this report summarizes the individualized responses to the different types of attacks. These responses are also listed on a separate, one-page, foldable card that notes: what the victim might experience; an overarching goal; and a list of four-five response actions for each terror attack scenario.

Chapter Four concludes by reasserting the goals of the project; noting that a proper dissemination strategy is not part of the research; and third, nesting the work in a larger body of work relating to risk assessment - specifically, applying a risk assessment framework to individual decisionmaking for people who may find themselves struggling to survive a catastrophic terror event.

As a critique, it is hard to gauge the ultimate efficacy of an individualized terror event survival strategy per se, especially when proper dissemination is difficult to attain. Moreover, many of the strategic suggestions rest on public disaster preparedness. It is noted that in biological attacks the individual's knowledge of such an event is limited and delayed; thus, public preparedness efforts are crucial to an individual's survival. This also goes for chemical, radiological and nuclear events, where limiting one's exposure to these agents is necessary to survive. Thus, evacuation plans are in order, with these also falling under larger public response organizations. That said, the author's efforts at separating out potential catastrophic terror events and their specific survival strategies is a noble one.

The second report to be covered here is on the vulnerability of the US food supply to terror attacks. It is entitled: Hitting America's Soft Underbelly: The Potential Threat of Deliberate Biological Attacks Against the U.S. Agricultural and Food Industry, by Peter Chalk. This research, funded by the Office of the Secretary of Defense, is organized into four chapters: introduction; vulnerabilities in the food sectors; potential impact of an 'agro-terror' event; and policy recommendations.

Aside from the obvious importance of food for a population to survive, the US agriculture and food sectors account for just over 12 per cent of the workforce, and constitute the nation's largest export industry. The author points out that this industry is also vulnerable to large-scale disruption due to its concentrated and intensive production operations and to a lack of security focus over the years. Add to that the potential for diseased livestock-and a lack of research focus in this area-and the result is a soft and critical sector to target. The author illustrates the tremendous costs of managing an agro-terror event targeting livestock: the direct costs associated with quarantining and destroying large stocks of animals; a multiplier cost related to compensating farmers for destroyed stock; and the indirect cost of raised tariffs on US exports due to fears of native infestation.

In addition to the critical nature of the industry to the US economy, it is also vulnerable, with 15 biologically hardly pathogens recognized as severely disrupting livestock health. The author points to the foot and mouth disease outbreak in Britain in 2001, which resulted in 3.5 million 
euthanized animals. Despite this vulnerability, there have been only 12 documented cases of intentional biological attacks against animals since 1912, with only two of these events having some link to a terror goal.

In his final chapter, the author makes a number of policy suggestions. Those germane to the field of security include an increased focus on intelligence measures, bio-security and surveillance, and better education and training for forensic investigators to ascertain whether a disease outbreak was the result of a terror event. These policy suggestions are offered to focus more attention on these issues in public policy circles, with an obvious call for more diligence and resources directed towards the nation's food security.

This report is part of a larger effort to ascertain the criticality and vulnerability of major US industries and sectors post-9/11. With air travel getting the brunt of resources, many researchers have turned to a diverse group of industries that are both critical and vulnerable: these include chemical plants, port facilities, water supplies and, here, the nation's food supply.

These two reviews hopefully will draw practitioners and educators to explore the resources evident at research centers like Rand and government agencies in the US such as the National Institute of Justice (at www.nij.gov) and the US Department of Homeland Security (at www.dhs.gov/dhspublic theme_home5.jsp), as well as the British Home Office (at www.homeoffice.gov.uk/terrorism/ index.html), that supply policy-relevant security research.

Robert Stokes

L. Douglas Wilder School of Government and Public Policy

Virginia Commonwealth University 